\title{
GCU
}

Glasgow Caledonian

University

University for the Common Good

\section{Abnormal photoreceptor outer segment development and early retinal degeneration in kif3a mutant zebrafish}

Raghupathy, Rakesh K.; Zhang, Xun; Alhasani, Reem H.; Zhou, Xinzhi; Mullen, Margaret; Reilly, James; Li, Wenchang; Liu, Mugen; Shu, Xinhua

Published in:

Cell Biochemistry and Function

DOI:

$10.1002 /$ cbf.3205

Publication date:

2016

Document Version

Author accepted manuscript

Link to publication in ResearchOnline

Citation for published version (Harvard):

Raghupathy, RK, Zhang, X, Alhasani, RH, Zhou, X, Mullen, M, Reilly, J, Li, W, Liu, M \& Shu, X 2016, 'Abnormal photoreceptor outer segment development and early retinal degeneration in kif3a mutant zebrafish', Cell

Biochemistry and Function, vol. 34, no. 6, pp. 429-440. https://doi.org/10.1002/cbf.3205

\section{General rights}

Copyright and moral rights for the publications made accessible in the public portal are retained by the authors and/or other copyright owners and it is a condition of accessing publications that users recognise and abide by the legal requirements associated with these rights.

Take down policy

If you believe that this document breaches copyright please view our takedown policy at https://edshare.gcu.ac.uk/id/eprint/5179 for details

of how to contact us. 
Abnormal photoreceptor outer segment development and early retinal degeneration in kif $3 a$ mutant zebrafish

Rakesh K. Raghupathy ${ }^{1 \#}$, Xun Zhang ${ }^{1 \#}$, Reem H. Alhasani ${ }^{1}$, Xinzhi Zhou ${ }^{1}$, Margaret Mullin ${ }^{2}$, James Reilly ${ }^{1}$, Wenchang $\mathrm{Li}^{3}$, Mugen $\mathrm{Liu}^{4}$, Xinhua Shu ${ }^{1, *}$

1 Department of Life Sciences, Glasgow Caledonian University, Glasgow G4 0BA, UK

2 School of Life Sciences, University of Glasgow, Glasgow G12 8QQ, UK

3 School of Psychology and Neuroscience, University of St Andrews, St Andrews KY16 9AJ, UK

4 Key Laboratory of Molecular Biophysics of Ministry of Education, College of Life Science and

Technology, Center for Human Genome Research, Huazhong University of Science and

Technology, Wuhan, Hubei 430074, PR China

\#The authors have contributed equally to the work.

*Corresponding author: Xinhua Shu, Department of Life Sciences, Glasgow Caledonian University, Cowcaddens Road, Glasgow, UK, E-mail: Xinhua.Shu@gcu.ac.uk 


\begin{abstract}
Photoreceptors are highly specialized sensory neurones that possess a modified primary cilium called the outer segment. Photoreceptor outer segment formation and maintenance require highly active protein transport via a process known as intraflagellar transport (IFT). Anterograde transport in outer segments is powered by the heterotrimeric kinesin-II and coordinated by IFT proteins. Here we describe a new zebrafish model carrying a nonsense mutation in the kinesin-II family member 3A (kif3a) gene. Kif3a mutant zebrafish exhibited curved body axes and kidney cysts. Outer segments were not formed in most parts of the mutant retina and rhodopsin was mislocalized, suggesting KIF3A has a role in rhodopsin trafficking. Both rod and cone photoreceptors degenerated rapidly between 4 and 9 days post fertilization (dpf) and electroretinography response was not detected in 7 dpf mutant larvae. Loss of KIF3A in zebrafish also resulted in an intracellular transport defect affecting anterograde but not retrograde transport of organelles. Our results indicate KIF3A plays a conserved role in photoreceptor outer segment formation and intracellular transport.
\end{abstract}

Keywords Zebrafish; Kif3a; outer segment; anterograde transport; retinal degeneration 


\section{INTRODUCTION}

Primary cilia are organelles that protrude from the cell surface and serve as cell surface signaling centres during embryogenesis and tissue homeostasis. ${ }^{1}$ The assembly and maintenance of primary cilia require the intraflagellar transport (IFT) machinery. IFT particles are responsible for the bidirectional movement of ciliary cargos along the axoneme. IFT proteins form two complexes: complex A and complex B. Vertebrate IFT complex A contains at least 6 proteins while more than 10 different proteins are in complex B. ${ }^{2}$ IFT complex A mediates retrograde transport powered by the dynein motor protein. IFT complex B organizes anterograde transport toward the ciliary tip driven by the motor proteins, heterotrimeric Kinesin II and homodimeric KIF17. Kinesin II comprises two motor subunits, KIF3A and KIF3B (or KIF3C) and the Kinesin associated protein 3 (KAP3). ${ }^{3-4}$ Previous reports showed that Kinesin II and KIF17 are both involved in vertebrate ciliogenesis. ${ }^{5-7}$ Photoreceptor outer segments are modified sensory cilia. Outer segments contain packed membranes and membrane-associated proteins that participate in phototransduction. Proteins are synthesized in the inner segments and transported to the outer segments through the narrow connecting cilium at high flow rates. ${ }^{8}$ The development and maintenance of outer segments depends on the IFT machinery. ${ }^{9}$ Kinesin II motor subunits and IFT were reported to be associated with photoreceptor axonemes ${ }^{10-11}$ and to be co-immunoprecipitated in retinal extracts. ${ }^{12}$ Deficiency of Kinesin II motor subunits or IFT components in mouse and zebrafish caused abnormal photoreceptors and retinal degeneration. ${ }^{9}$ Conditional deletion of Kif3a in mouse photoreceptor cells led to the mislocalization of opsin and arrestin, but not peripherin or transducin, implicating selective mechanisms for anterograde transport. ${ }^{11}$ The conditional knockout (KO) mice also displayed rapid photoreceptor cell death, possibly through mislocalized opsin triggering apoptosis. ${ }^{11,13}$ In another study involving conditional KO mice (in which Kif3a was deleted in rod or cone cells), Kif3a appeared to play a different role in each type of photoreceptor; it was required for ciliary protein trafficking in cone cells but not in rod cells, though Kif3a KO rod cells rapidly degenerated. ${ }^{14}$ Global knock-out of Kif3b in mouse also caused embryonic lethality. ${ }^{6}$ However, depletion of Kif3c did not result in any obvious abnormality. ${ }^{15-}$ 16 
Zebrafish have been used to investigate the function of the kinesin II family in cilia. Previous reports showed morpholino knockdown of zebrafish Kif17 resulted in disruption of photoreceptor outer segment formation and mislocalization of photoreceptor membrane proteins. ${ }^{7}$ The same group carried out a morpholino knockdown of kif $3 b$ in zebrafish and found that the kif $3 b$ morphants exhibited an early embryonic defect. When dominant-negative kif3b or kif 17 mutants were expressed in zebrafish embryonic cone cells, this resulted in abnormal cone development. ${ }^{17}$ Recently, Zhao et al characterized an N-ethyl-N-nitrosourea (ENU) induced zebrafish mutant, which carried a nonsense mutation in the kif3b gene. ${ }^{18}$ Kif $3 b$ mutant zebrafish exhibited a downward body curvature, indicating a ciliary defect. Photoreceptor outer segment formation was delayed and the outer segments that formed were shorter and showed an abnormal shape, suggesting that Kif3b has a role in outer segment formation. In the mutant zebrafish, rod cells degenerated between 3-5 dpf; the cone cells, however, developed normal outer segments and survived longer, indicating that Kif3b functions differently in rod and cone cells. Morpholino knockdown of kif3c in zebrafish did not cause any ciliary defect. However, knockdown of Kif3c in Kif3b mutants resulted in absence of photoreceptor, indicating that Kif3c functions redundantly with Kif3b. ${ }^{18}$ A nonsense kif17 zebrafish mutant was also characterized but did not display any obvious ciliary defect in the examined tissues (retina, ear, spinal cord and pronephric duct) except the nasal cilia, which were shorter. Rod and cone opsins were not mislocalized in the kif17 mutants, suggesting Kif17 is not involved in photoreceptor ciliary trafficking. ${ }^{18}$ Recently an ENU-induced mutant kif3a zebrafish, which had two additional amino acids (MQ) within the motor domain of KIF3A, was reported to exhibit short cilia and kidney cysts; however, the retina of the mutant zebrafish was not phenotypically analysed. ${ }^{19}$ To address the question of whether loss of Kif3a can cause a defect in photoreceptor protein trafficking and retinal degeneration in zebrafish, we characterized an ENU-induced kif3a nonsense mutant zebrafish line. Our results indicate that deletion of KIF3A resulted in abnormal development of photoreceptor outer segments, mislocalization of rhodopsin and rapid photoreceptor degeneration. 


\section{MATERIALS AND METHODS}

\section{Zebrafish maintenance}

Zebrafish were bred and maintained according to the procedures described by Westerfield. ${ }^{20}$ Embryos were cultured in E3 medium in an incubator at $28^{\circ} \mathrm{C}$. The Kif $3 a^{S A 1617}$ mutant zebrafish was provided by the Wellcome Trust Sanger Institute. All animal work was carried out in accordance with the UK Animals (Scientific Procedures) Act, 1998 and associated guidance.

\section{Alignment of KIF3A protein sequences}

KIF3A peptide sequences of zebrafish and other species were obtained from Ensembl.org database and were aligned using Clustal Omega software (http://www.ebi.ac.uk/). Conserved domains were box-shaded using the BoxShade 3.21 software (http://www.ch.embnet.org/).

\section{Measurement of eye size}

Wildtype and the $K i f 3 a^{S A 1617}$ mutant embryos at 2, 3, 4, 5 and 6 dpf were anaesthetised with Tracine and photographed. Photo prints were used for eye measurement. Eye measurements were taken from the anterior to the posterior edge. 40 eyes of wildtype or Kif $3 a^{S A 1617}$ mutant embryos at each age point were measured.

Reverse transcription polymerase chain reaction (RT-PCR) and quantitative real-time PCR Total RNA was extracted from zebrafish embryos and adult tissues with the Trizol Reagent (Sigma). cDNAs were synthesized using a Transcriptor High Fidelity cDNA synthesis kit (Roche). The cDNA was amplified using a NEB standard Taq polymerase system with the primers ZFKIF3AF2 5'AGGAGATCTCGGGCTCTGA3' and ZFKIF3AR2 5'CTCCTCAGGCTTAGCCAATAAA3'. A 517-bp fragment of $\beta$-actin was amplified as a control for PCR using forward primer 5'TGCCATGTATGTGGCCATCCA3' and reverse primer 5'ACCTCCAGACAGCACTGTGT3'. PCR products were analysed by agarose gel electrophoresis. To quantify the expression of kif $3 a$ in the Kif $3 a^{S A 1617}$ mutant and wildtype zebrafish, RNA from 10 wildtype (pooled) or 10 mutant (pooled) zebrafish at $5 \mathrm{dpf}$ was extracted and $\mathrm{cDN}$ was synthesized. A quantitative real-time PCR was carried 
out using platinum sybr green qPCR supermix (Thermo Fisher Scientific, UK) with the primers, ZFKIF3AF2 and ZFKIF3AR2. Expression of zebrafish 18S RNA gene was used for normalization; the primers used for $18 \mathrm{~S}$ rRNA gene were 5'CCACTCCCGAGATCAACTA3' and 5'CAAATTACCCATTCCCGACA3'. The amount of kif3a mRNA is determined by normalizing the threshold cycle $C_{\mathrm{T}}$ of kif3a gene to the $C_{\mathrm{T}}$ of zebrafish $18 \mathrm{~S}$ rRNA gene in the same sample based on the following formula: the average $C_{\mathrm{T}}$ of kif $3 a$ gene minus the average $C_{\mathrm{T}}$ of $18 \mathrm{~S}$ rRNA gene in which this result is recognized as $\Delta C_{\mathrm{T}}$ where it is specific for each gene and can be compared with $\Delta C_{\mathrm{T}}$ of calibration samples. The difference between $\Delta C_{\mathrm{T}}$ of wildtype and $K i f 3 a^{S A 1617}$ mutant zebrafish is known as $\Delta \Delta C_{\mathrm{T}}$. The relative quantification of kif3a expression in Kif $3 a^{S A 1617}$ mutant zebrafish is calculated in comparison with wildtype zebrafish according to the following formula: $2^{-\triangle \triangle C T}$ and represented as a fold change.

\section{Genotyping}

The zebrafish were anaesthetised and a small portion of the tail fin was clipped. DNA was extracted from the fin clipping by adding $25 \mu 1$ of methanol and incubating at room temperature for 5 minutes. $15 \mu 1$ of TE-Tween $20(10 \mathrm{mM}$ Tris, $1 \mathrm{mM}$ EDTA, $\mathrm{pH} 8.0,0.33 \%$ Tween-20) and $0.5 \mu 1$ of $10 \mathrm{mg} / \mathrm{ml}$ proteinase $\mathrm{K}$ were added to the sample and incubated at $56^{\circ} \mathrm{C}$ for at least 2 hours in a thermal cycler machine. The tube was then incubated at $98^{\circ} \mathrm{C}$ for 10 minutes to inactivate the proteinase $\mathrm{K} .45 \mu 1$ of $\mathrm{dH}_{2} \mathrm{O}$ was added to the sample and mixed well. 200ng of the extracted DNA was used as template in $20 \mu 1$ PCR reaction to amplify the DNA fragment by PCR with the primers, ZFKIF3AF1: 5' AGGAGATCTCGGGCTCTGA3' and ZFKIF3AR1: 5'CACATGCTGCCTCATGTTTC3'. PCR products were analysed by agarose gel electrophoresis and sequenced to determine the genotype.

\section{Histology and transmission electron microscopy}

For histological analyses the wildtype and $\mathrm{Kif}_{3} a^{\mathrm{SAl617}}$ mutant zebrafish at 3, 5, 6, and 9 dpf were fixed in 4\% paraformaldehyde, dehydrated, embedded in paraffin and sectioned in accordance with previous descriptions. ${ }^{21}$ The sections were dewaxed and subjected to haematoxylin and eosin staining. 
For retinal ultrastuctural analyses, the wildtype and $K i f 3 a^{S A 1617}$ mutant fish at $4 \mathrm{dpf}$ and $5 \mathrm{dpf}$ were fixed and embedded as described previously. ${ }^{22}$ Briefly, embryos were fixed in $4 \%$ glutaraldehyde, $1 \%$ paraformaldehyde in $0.1 \mathrm{M}$ sodium cacodylate buffer and secondarily fixed with $1 \%$ osmium tetroxide in $0.1 \mathrm{M}$ sodium cacodylate buffer, followed by dehydration through a series of ethanol solutions and infiltration in a 1:1 PO:EPON resin mix. Ultrathin sections of 50-60nm thickness were cut using a Leica UTC Ultratome, collected on 100 mesh formvar-coated copper grids and contrast stained with $2 \%$ uranyl acetate and Reynolds lead citrate. The sections of zebrafish eyes were viewed on a Tecnai T20 transmission electron microscope and images were captured using Gatan Digital imaging software.

\section{Immunohistochemistry}

Sections from 4\% paraformaldehyde fixed and paraffin embedded 5dpf wildtype and $\mathrm{Kif}_{3} \mathrm{a}^{\mathrm{SAl617}}$ mutant were dewaxed and washed with PBS, immersed in antigen retrieval buffer (10mM Tris Base, $1 \mathrm{mM}$ EDTA, $0.05 \%$ Tween 20, $\mathrm{PH}=9.0$ ) and boiled for $20 \mathrm{~min}$. The sections were blocked in $1 \times \mathrm{TBS} / 0.3 \%$ Triton $\mathrm{X}-100 / 5 \%$ sheep serum for 1 hour at room temperature and then incubated with primary antibodies diluted in blocking solution overnight at $4^{\circ} \mathrm{C}$. The primary antibodies included mouse anti-ZPR1 (1:200, ZIRC, USA), mouse anti-rhodopsin 4D2 (1:200, Abcam, UK), antiacetylated alpha tubulin antibody and rabbit anti-KIF3A (1:50, Abcam, UK) antibodies. After washing, the sections were incubated with FITC-conjugated secondary antibody at 1:400 dilution. The nuclei were counterstained with DAPI and images were captured using confocal microscopy (LSM 800, Carl Zeiss Inc.).

\section{Detection of cell death}

Apoptotic cells in the retinas of Kif $3 a^{S A 1617}$ mutant zebrafish at $5 \mathrm{dpf}$ were detected by the DeadEnd $^{\mathrm{TM}}$ Fluorometric TUNEL System (Promega, Madison, USA) according to the manufacturer's instruction. The zebrafish samples were fixed and cryosectioned as described above. The cryosections of zebrafish eyes were washed with $1 \times \mathrm{PBS}$ for $5 \mathrm{~min}$ before being fixed again by $4 \%$ PFA/PBS. After permeabilisation with $20 \mu \mathrm{g} / \mathrm{ml}$ proteinase $\mathrm{K}$ solution, the sections were 
labelled with $\mathrm{rTdT}$ reaction mix for 1 hour at $37^{\circ} \mathrm{C}$ and the reaction was stopped with $2 \times \mathrm{SSC}$. Slides were mounted with DAPI and images were captured using ZEISS LSM 800 confocal microscopy. In order to quantify cell death, the number of TUNEL positive cells in a sample of 500 cells of the outer nuclear layer was counted from five slides (100 cells in each slide from each fish sample) of wildtype and mutant retinas respectively.

\section{Electroretinography}

Electroretinography (ERG) was performed under photopic condition according to previous descriptions. ${ }^{23}$ Briefly, $7 \mathrm{dpf}$ larvae were anesthetized in tricaine and placed on the top of a wet filter tissue. A silver wire was placed beneath the larvae as the reference electrode and a glass electrode was positioned onto the cornea. ERG recording was made using an Axon Multiclamp 700B amplifier. Signals were band-filtered between 3 and $100 \mathrm{~Hz}$.

\section{Melanosome transport assay}

Kif $3 a^{S A 1617}$ mutants and siblings at 4dpf were exposed to epinephrine (500 $\mu \mathrm{g} / \mathrm{ml}$, Sigma) in E3 medium. Melanosome retraction was continuously monitored under microscopy and the endpoint was scored when all melanosomes in the head and trunk were perinuclear. ${ }^{21}$ To evaluate the melanosome dispersion, larvae were washed with excess E3 medium after epinephrine exposure and exposed to caffeine (1mg/ml, Sigma) in E3 medium. The dispersion was also monitored and the endpoint scored. Images were taken throughout the monitoring processes.

\section{Statistical analysis}

Statistical analysis was carried out using the Graphpad Prism6 software. All data were presented as the mean $\pm \mathrm{SD}$. Statistical significance was analyzed using unpaired Student's $t$ test.

\section{RESULTS}

Expression of Kif3a during zebrafish development and in adult tissues 
The zebrafish Kif3a gene contains 17 exons and encodes a 701 amino acid polypeptide. The KIF3A protein is highly conserved across species; protein sequence comparison showed zebrafish KIF3A is 92.5\% identical to human KIF3A, $91.8 \%$ to mouse, $92.1 \%$ to chicken, $91.2 \%$ to Xenopus and $56.5 \%$ to C. elegans (Supplementary Materials, Fig. S1).

We used RT-PCR to analyse the temporal and spatial expression patterns of Kif3a gene expression during zebrafish embryogenesis. The Kif3a transcript was readily detected in oocytes and during embryogenesis throughout the larval stages (Fig. 1A). Kif3a expression in adult tissues was examined in total RNA extracted from eye, brain, heart, testis, liver, kidney, ovary, intestine, muscle and skin by RT-PCR. The Kif3a transcript was readily detected in the eye, ovary, muscle and liver; it was also detectable in testis, brain and heart, and weakly in kidney, skin and intestine (Fig. 1B).

\section{Kif $3 a^{\text {SA1617 }}$ mutant zebrafish}

A zebrafish Kif3 ${ }^{S A 1617}$ mutant was produced by N-ethyl-N-nitrosourea (ENU) mutagenesis, carrying a nonsense mutation at position 1363 changing cytosine to thymine $(\mathrm{C}>\mathrm{T})$ in the exon 9 of the Kif $3 a$ gene (NM_001017604.2). The change $1363 \mathrm{C}>\mathrm{T}$ is predicted to cause an Arginine (CGA) to a TGA stop codon in the rod domain of zebrafish KIF3A, resulting in a truncated polypeptide of 409aa instead of 701aa (NP_001017604.2) (Fig. 2A-C). The nonsense mutation was predicted to lead to nonsense-mediated decay (NMD). Quantitative real-time PCR revealed that kif3a mRNA was decreased by about $88 \%$ in 5 dpf Kif $3 a^{\text {SAl617 }}$ mutant larvae when compared to wildtype siblings $(\mathrm{p}=0.0065)$, confirming the occurrence of NMD (Supplementary Materials, Fig. S2). We also used a polyclonal antibody to immunostain retinal sections of $5 \mathrm{dpf}$ wildtype and mutant siblings and found that the KIF3A protein was localized to the connecting cilia of wildtype photoreceptor cells, consistent with previous reports of KIF3A localization in the photoreceptors of various species. ${ }^{10,24,25}$ However, KIF3A expression was not detected in the photoreceptors of $5 \mathrm{dpf} \mathrm{Kif}_{3} \mathrm{a}^{\mathrm{SAl617}}$ mutant retinas (Fig. 2D), suggesting that $K i f 3 a^{S A 1617}$ is a null allele. When the retinal sections were stained with antiacetylated tubulin antibody, connecting cilia were not appeared in the $\mathrm{Kif}_{3} a^{\mathrm{SAl617}}$ mutant (Fig. 2D).

Kif $3 a^{S A 1617}$ mutants exhibited curved body axes and kidney cysts, which are major features of ciliary defects (Fig. 3). Kidney cyst formation was evident at $72 \mathrm{hpf}$ and became more pronounced by 
4dpf. The eyes of both wildtype and Kif $3 a^{S A 1617}$ mutant zebrafish kept growing, while the mutants had smaller eyes than that of wildtype zebrafish measured at age 2, 3, 4, 5 and $6 \mathrm{dpf}$, respectively (Supplementary Materials, Fig. S3). The mutant zebrafish survived at most until 15dpf with death resulting from multiple organ dysfunctions, e.g. the kidney cyst.

\section{Loss of Kif3a resulted in abnormal photoreceptor outer segment development}

Cone-specific knock-out of Kif $3 a$ in mouse has been reported to cause misaligned cone outer segment membranes at P13 [14]. More recently, depletion of Kif3a in mouse retinal progenitors was shown to cause the absence of outer segment morphogenesis. ${ }^{26}$ We therefore asked whether there was evidence of developmental defects in the outer segments of Kif $3 a^{\text {SAl617 }}$ mutant retinas. Using transmission electron microscopy we examined the ultrastructure of both wildtype and Kif $3 a^{S A 1617}$ mutant outer retinas at $4 \mathrm{dpf}$ and $5 \mathrm{dpf}$. Photoreceptors in wildtype larvae exhibited well-formed elongated outer segments (Fig. 4A-1 and B-1), while in 4 dpf mutant larvae the outer segments were absent in most parts of the retina. The outer segments remaining in the peripheral retina displayed abnormal shapes, including distended outer segment with folded protrusion, annular outer segment, irregular branching of outer segments and compressed and distended outer segments (Fig. 4A-2, 3, 4, 5, 6). In mutants at $5 \mathrm{dpf}$, outer segments were still not observable in most parts of the retina, although in the peripheral retina abnormal outer segments (including vestigial, annular, delaminated and warped with irregular delamination) were present but had started to lose structural integrity (Fig. 4B-2, 3, 4, 5, 6).

\section{Early rapid retinal degeneration in Kif $3 a^{\text {SA1617 }}$ mutant zebrafish}

As abnormal outer segment development is associated with retinal degeneration in mouse and zebrafish, ${ }^{27,28}$ we asked whether there is retinal degeneration in Kif $3 a^{\text {SA1617 }}$ mutants. We examined histological sections of both wildtype and Kif $3 a^{S A 1617}$ mutants by light microscopy. Both wildtype and mutants at 3 dpf exhibited three well-formed layers (outer nuclear layer, inner nuclear layer and ganglion cell layer), suggesting that Kifßa is not involved in retinal lamination. There was no morphological difference in the outer nuclear layers between wildtype and mutant zebrafish at $3 \mathrm{dpf}$. At $5 \mathrm{dpf}$, the mutants displayed a thinner outer nuclear layer when compared to that of wildtype 
zebrafish. The degeneration of photoreceptors was clearly evident in the central part of mutant retinas at $6 \mathrm{dpf}$, but photoreceptors in the periphery were less affected. All photoreceptors in the mutant were degenerated by $9 \mathrm{dpf}$ and the outer nuclear layer had disappeared (Fig. 5).

We also examined how rod and cone cells were affected by using anti-rhodopsin antibody (4D2) and ZPR1 antibody to label rod outer segments and red and green double cones, respectively, in immunofluorescence analyses. The rod outer segments were well-formed in wildtype at $5 \mathrm{dpf}$. In the age-matched Kif $3 a^{S A 1617}$ mutants, however, the outer segments were absent, while rhodopsin was mislocalized to the rod inner segments and to the synapses in the peripheral area, almost all rods had disappeared in the central area (Fig. 6A). Meanwhile, the double cone cells labelled by ZRP1 antibody were shorter in the mutants while only a few cone cells remained in the central retina of the mutants (Fig. 6B), indicating that cone degeneration was also predominant in the central retina. To confirm the photoreceptor cell death, we carried out a TUNEL assay on retinal sections of both wildtype and Kif $3 a^{S A 1617}$ mutants at 5 dpf. TUNEL-positive nuclei were barely observed in wildtype retina but could be found in both central and peripheral retina in the mutants (Fig. 7). There were also some TUNEL-positive nuclei present in the inner nuclear layer of the mutants.

We also used ERG to test visual impairment in the Kif $3 a^{S A 1617}$ mutants. Zebrafish cone cells mature earlier than rod cells, so the ERG response of larvae at 5 and $7 \mathrm{dpf}$ is determined by cones. Loss of functional cones results in the absence of ERG. ${ }^{29,30}$ ERG was performed in 7 dpf wildtype and Kif $3 a^{S A 1617}$ mutant larvae. The wildtype zebrafish displayed normal responses, as expected, while the cone ERG response was absent in the mutant zebrafish (Supplementary Materials, Fig.S4).

\section{Kif $3 a^{\text {SA1617 }}$ mutants exhibited delayed anterograde intracellular transport}

In response to light or drugs, zebrafish can alter their skin pigmentation by promoting pigment granule aggregation and dispersion. ${ }^{21,31}$ The pigment granules (melanosomes) are lysosome-related granules and their bi-directional shuttling towards the perinuclear region (aggregation) or toward the cell periphery (dispersion) is driven by different motor proteins. Kinesin II is responsible for dispersion of melanosomes, whereas melanosome aggregation requires cytoplasmic dynein. ${ }^{31}$ Pigment 
dispersion (anterograde) or aggregation (retrograde) can be stimulated within minutes after treatment with caffeine and epinephrine, respectively. ${ }^{31}$

In our assay, $4 \mathrm{dpf}$ embryos were dark-adapted to display melanophore dispersion. Once the embryos were exposed to epinephrine, the melanosomes rapidly contracted and the pigmentation area was reduced. At the maximum aggregation endpoint all the pigment cells exhibited perinuclear accumulation of melanosomes (Fig. 8). We measured the time required for reaching the pigment aggregation endpoint and did not find any difference between wildtype (3.84 min) and the Kif $3 a^{S A 1617}$ mutants $(3.73 \mathrm{~min})$. We then evaluated anterograde melanosome transport by measuring the recovery time to reach full pigment dispersion after embryos were treated with caffeine. The wildtype embryos rapidly dispersed their melanosomes (3.5 min) whereas the Kif $3 a^{S A 1617}$ mutants showed significantly delayed melanosomes dispersion (15.765 min, $\mathrm{p}<0.0001)$ (Fig. 8).

\section{DISCUSSION}

In this study we examined the phenotypes of a newly identified zebrafish mutant, Kif $3 a^{\text {SA1617 }}$, which carries a nonsense mutation in the Kif $3 a$ gene. Kif $3 a^{S A 1617}$ mutant zebrafish did not form outer segments in most parts of the retina, although some abnormal outer segments were formed in the peripheral retina. The first photoreceptor-specific Kif $3 a$ knockout mouse model was made using an IRBP-Cre transgene and Cre-loxP, in which the Kif3a gene was excised in the third postnatal week. ${ }^{11}$ The outer segments were formed in this model. When a Rho (rhodopsin)-Cre transgene was used to knockout the Kif3a gene in rod cells, the uniform excision of Kif $3 a$ gene across the whole retina was visible by the second postnatal week. The mouse model also exhibited changes in outer segment formation. ${ }^{32}$ Cone-specific knockout of Kif3a in mouse using Cre together with the human red-green opsin (Hrgp) promoter did not affect cone outer segment formation, but the outer segment membranes appeared misaligned. ${ }^{14}$ However, a recent mouse model made with a Six3-Cre transgenes in which Kif3a was deleted in photoreceptor progenitors by embryonic day 9.5 , had no outer segment formation. ${ }^{26}$ The data suggest that the KIF3A protein remained functional for outer segment formation in the three Kif3a knockout mouse models generated using IRBP-Cre, Rho-Cre or Hrgp-Cre. ${ }^{11,14,32}$ 
KIF3A may act alone to drive anterograde transport in zebrafish photoreceptors, since loss of Kif17 did not cause any morphological defect in zebrafish photoreceptors. ${ }^{18} \mathrm{KIF} 3 \mathrm{~A}$ is involved in the transport of the IFT subcomplex B, which contains IFT20, 27, 46, 52, 57, 74/72, 80, 81, 88 and $172 .^{2}$ Null mutations in zebrafish ift57, ift88 or ift172 caused externally visible phenotypes (curved body axis, smaller eyes and kidney cyst formation) and retinal defects similar to that of $\mathrm{Kif}_{3} \mathrm{a}^{\mathrm{SAl617}}$ mutant zebrafish. ${ }^{33-36}$ Ift 88 and ift172 mutants failed to form outer segments, whereas ift57 mutants displayed shorter outer segments. ${ }^{33-35}$ Some types of cilia are initially able to form but degenerate over time in the ift zebrafish mutants, presumablysuggesting that ift is critical for cilia maintenance but not assembly. However, maternal-zygotic ift 88 , ift57and ift 172 mutants showed severely impaired cilia formation, suggesting ift is required for ciliogenesis. ${ }^{36,37}$ This is because the maternal contribution of IFT proteins is required for the genesis of some types of cilia. This also happens in Kif3b zebrafish mutants, in which Kupffer's vesicle formation is not affected, possibly due to the presence of a maternal contribution. ${ }^{18}$. Abnormal outer segments are formed in a small number of photoreceptors in the Kif $3 a^{S A 1617}$ mutant zebrafish (Fig. 4). This may also possibly be due to a maternal contribution.

Opsins are the most abundant protein component in photoreceptor outer segments. Opsins are synthesized in photoreceptor inner segments and transported to outer segments through anterograde trafficking driven by kinesin II motors. ${ }^{9}$ Any defect in the trafficking process results in opsin mislocalization and subsequent photoreceptor degeneration. Knockout of Kif3a in rod and/or cone photoreceptors results in rhodopsin mislocalization in rod cells and mistargeting of S- and M/L-opsins in cone cells. ${ }^{11,13,14,32}$ Opsin mislocalization has been observed in ift mutant mice and in zebrafish. ${ }^{27,33,34,35}$ Here we also showed opsin mislocalization in Kif3a mutant zebrafish, demonstrating that both Kif3a and IFT proteins are required for opsin trafficking. Live-cell analyses provided evidence that Kif3a is required for photoreceptor opsin transport. ${ }^{38}$ Opsin mislocalization has been proposed to trigger photoreceptor cell death and the level of mislocalized opsin is correlated with the speed of retinal degeneration. ${ }^{13,39,40}$ Kif3a knockout mouse models exhibited an early and rapid retinal degeneration. ${ }^{11,13,14,26,32}$ while a reduction of mislocalized opsin in Kif3a knockout mice was shown to ameliorate photoreceptor cell death. ${ }^{13}$ Photoreceptor cell death was also shown in the rapid retinal degeneration in Kif3a mutant zebrafish. Similarly, ift mutant zebrafish also exhibited an 
early and rapid retinal degeneration. ${ }^{33-35}$ The mechanism by which mislocalized opsin triggers cell death remains elusive, although previous in vitro work suggested that opsin mislocalization stimulated the heterotrimeric $\mathrm{G}$ protein to increase the activity of adenylate cyclase, which was proposed to produce more cAMP. ${ }^{41}$ An increase in cAMP level was observed in some retinal degeneration animal models and proposed to promote photoreceptor cell death. ${ }^{42}$

Kinesin II also plays an important role in the transport of membrane-bound organelles, including the pigment organelles. ${ }^{43}$ Fish melanophores contain hundreds of melanin-filled melanosomes and offer an outstanding system for studying intracellular transport. Melanophores can aggregate their melanosomes at the cell centre or disperse them throughout the cytoplasm, allowing visible colour changes in response to environment conditions. The function of KIF3A was further elucidated by the demonstration that its loss resulted in disruption of the anterograde (plus-end-directed) movement of skin melanosomes along microtubules but did not disrupt reterograde transport. Previous work in Xenopus demonstrated that the interaction between the dynactin subunit $\mathrm{p} 150^{\text {Glued }}$ and the KAP subunit of kinesin II was required for melanosome dispersion. ${ }^{44}$ A recent study showed that KIF3A also interacted with p150 Glued through its C-terminal cargo-binding domain (residues 365-701) and functioned in the organization of centriole subdistal appendages. ${ }^{45}$ Further work is required to investigate whether the interaction between KIF3A and $\mathrm{p} 150^{\text {Glued }}$ is involved in zebrafish melanosome dispersion.

Mouse and zebrafish models demonstrated that loss of KIF3A resulted in mislocalization of photoreceptor membrane proteins, including opsin, and in a rapid retinal degeneration. However the precise role of KIF3A in photoreceptor development and degeneration remains to be delineated. Our study provides a framework for dissecting the cellular function of KIF3A and elucidating the underlying mechanisms leading to the rapid retinal degeneration.

\section{CONFLICT OF INTEREST}

All authors certify that they have NO affiliations with or involvement in any organization or entity with any financial interest or non-financial interest in the subject matter or materials discussed in this manuscript. 


\section{AUTHOR CONTRIBUTIONS}

X.S. designed the study. R.K.R., X.Z., R.H.A., X.Z., M.M., W.L. performed the experiments. R.K.R., X.Z., R.H.A., and X.S. analysed the data, M.L., J.R. and X.S. wrote the main text. All authors reviewed the manuscript.

\section{ACKNOWLEDGEMENT}

The study was supported by the Royal Society of London, the National Eye Research Centre, the Visual Research Trust, the Fight for Sight, The Rosetrees Trust and the Yorkhill Children's Charity. XZ was funded by a Fight for Sight PhD studentship. RHA was funded by a Saudi Arabia government $\mathrm{PhD}$ scholarship. The maintenance of GCU zebrafish facility was funded by the EU INTERREG NEW noPILLS programme.

\section{REFERENCES}

1. Goetz SC, Anderson KV. The primary cilium: a signalling centre during vertebrate development. Nat. Rev. Genet. 2010; 11: 331-344.

2. Scholey JM. Intraflagellar transport motors in cilia: moving along the cell's antenna. J. Cell Biol. 2008; 180: 23-29.

3. Yamazaki H, Nakata T, Okada Y, Hirokawa N. KIF3A/B: a heterodimeric kinesin superfamily protein that works as a microtubule plus end-directed motor for membrane organelle transport. J. Cell Biol. 1995; 130: 1387-1399.

4. Muresan V, Abramson T, Lyass A, Winter D, Porro E, Hong F, Chamberlin NL, Schnapp BJ. KIF3C and KIF3A form a novel neuronal heteromeric kinesin that associates with membrane vesicles, Mol. Biol. Cell. 1998; 9: 637-652.

5. Marszalek JR, Ruiz-Lozano P, Roberts E, Chien KR, Goldstein LS. Situs inversus and embryonic ciliary morphogenesis defects in mouse mutants lacking the KIF3A subunit of kinesin-II. Proc. Natl. Acad. Sci. U. S. A. 1999; 96: 5043-5048. 
6. Nonaka S, Tanaka Y, Okada Y, Takeda S, Harada A, Kanai Y, Kido M, Hirokawa N.

Randomization of left-right asymmetry due to loss of nodal cilia generating leftward flow of extraembryonic fluid in mice lacking KIF3B motor protein. 1998; Cell 95: 829-837.

7. Insinna C, Pathak N, Perkins B, Drummond I, Besharse JC. The homodimeric kinesin, Kif17, is essential for vertebrate photoreceptor sensory outer segment development. Dev. Biol. 2008; 316: 160170.

8. Wolfrum U, Schmitt A. Rhodopsin transport in the membrane of the connecting cilium of mammalian photoreceptor cells. Cell Motil. Cytoskeleton 2000; 46: 95-107.

9. Insinna C, Besharse JC. Intraflagellar transport and the sensory outer segment of vertebrate photoreceptor. Develop. Dynamics 2008; 237: 1982-1992.

10. Whitehead JL, Wang SY, Bost-Usinger L, Hoang E, Frazer KA, Burnside B. Photoreceptor localization of the KIF3A and KIF3B subunits of the heterotrimeric microtubule motor kinesin II in vertebrate retina, Exp. Eye Res. 1999; 69: 491-503.

11. Marszalek JR, Liu X, Roberts EA, Chui D, Marth JD, Williams DS, Goldstein LS. Genetic evidence for selective transport of opsin and arrestin by kinesin-II in mammalian photoreceptors. Cell 2000; 102: 175-187.

12. Baker SA, Freeman K, Luby-Phelps K, Pazour GJ, Besharse JC. IFT20 links kinesin II with a mammalian intraflagellar transport complex that is conserved in motile flagella and sensory cilia. J. Biol. Chem. 2003; 278: 34211-34218.

13. Lopes VS, Jimeno D, Khanobdee K, Song X, Chen B, Nusinowitz S, Williams DS. Dysfunction of heterotrimeric kinesin- 2 in rod photoreceptor cells and the role of opsin mislocalization in rapid cell death. Mol. Biol. Cell 2010; 21: 4076-4088.

14. Avasthi P, Watt CB, Williams DS, Le YZ, Li S, Chen CK, Marc RE, Frederick JM, Baehr W. Trafficking of membrane proteins to cone but not rod outer segments is dependent on heterotrimeric kinesin-II. J Neurosci. 2009; 29: 14287-14298.

15. Yang Z, Roberts EA, Goldstein LS. Functional analysis of mouse kinesin motor Kif3C. Mol Cell Biol. 2001; 21: 5306-5311. 
16. Jimeno D, Lillo C, Roberts EA, Goldstein LS, Williams DS. Kinesin-2 and photoreceptor cell death: requirement of motor subunits. Exp. Eye Res. 2006; 82: 351-353.

17. Insinna C, Humby M, Sedmak T, Wolfrum U, Besharse JC. Different roles for KIF17 and kinesin II in photoreceptor development and maintenance. Dev. Dyn. 2009; 238: 2211-2222.

18. Zhao C, Omori Y, Brodowska K, Kovach P, Malicki J. Kinesin-2 family in vertebrate ciliogenesis, Proc. Natl. Acad. Sci. U. S. A.. 2012;109: 2388-2393.

19. Ryan S, Willer J, Marjoram L, Bagwell J, Mankiewicz J, Leshchiner I, Goessling W, Bagnat M, Katsanis N. Rapid identification of kidney cyst mutations by whole exome sequencing in Zebrafish, Development 2013; 140: 4445-4451.

20. Westerfield M. The Zebrafish Book, Eugene, OR: University of Oregon Press, 1995.

21. Shu X, Zeng Z, Gautier P, Lennon A, Gakovic M, Patton EE, Wright AF. Zebrafish RPGR is required for normal retinal development and plays a role in dynein-based retrograde transport processes. Hum. Mol. Genet. 2010; 19: 657-670.

22. Akhtar S, Patnaik SR, Raghupathy RK, Al-Mubrad TM, Craft JA, Shu X. Histological characterization of the Dicer1 mutant zebrafish retina. J. Ophthalmol. 2015; 2015: 309510.

23. Makhankov YV, Rinner O, Neuhauss SC. An inexpensive device for non-invasive electroretinography in small aquatic vertebrates. J. Neurosci. Methods 2004; 135 : 205-210. 24. Beech PL, Pagh-Roehl K, Noda Y, Hirokawa N, Burnside B, Rosenbaum JL. Localization of kinesin superfamily proteins to the connecting cilium of fish photoreceptors, J. Cell Sci. 1996; 109: 889-897.

25. Muresan V, Lyass A, Schnapp BJ. The kinesin motor KIF3A is a component of the presynaptic ribbon in vertebrate photoreceptors. J. Neurosci. 1999; $19: 1027-1037$.

26. Jiang L, Wei Y, Ronquillo CC, Marc RE, Yoder BK, Frederick JM, Baehr W. Heterotrimeric kinesin-2 (KIF3) mediates transition zone and axoneme formation of mouse photoreceptors. J. Biol. Chem. 2015; 290: 12765-12778.

27. Pazour GJ, Baker SA, Deane JA, Cole DG, Dickert BL, Rosenbaum JL, Witman GB, Besharse JC. The intraflagellar transport protein IFT88, is essential for vertebrate photoreceptor assembly and maintenance. J. Cell Biol. 2002; 157: 103-113. 
28. Bachmann-Gagescu R, Phelps IG, Stearns G, Link BA, Brockerhoff SE, Moens CB, Doherty D. The ciliopathy gene cc $2 \mathrm{~d} 2 \mathrm{a}$ controls zebrafish photoreceptor outer segment development through a role in Rab8-dependent vesicle trafficking. Hum. Mol. Genet. 2011; 20: 4041-4055.

29. Brockerhoff SE, Rieke F, Matthews HR, Taylor MR, Kennedy B, Ankoudinova I, Niemi GA, Tucker CL, Xiao M, Cilluffo MC, Fain GL, Hurley JB. Light stimulates a transducing-independent increase of cytoplasmic $\mathrm{Ca} 2+$ and suppression of current in cones from the zebrafish mutant nof. $\mathrm{J}$. Neurosci. 2003; 23: 470-480.

30. Stearns G, Evangelista M, Fadool JM, Brockerhoff SE. A mutation in the cone-specific pde6 gene causes rapid cone photoreceptor degeneration in zebrafish. J Neurosci. 2007; 27: 13866-13874. 31. Nascimento AA, Roland JT, Gelfand VI. Pigment cells: a model for the study of organelle transport, Annu. Rev. Cell Dev. Biol. 2003; 19: 469-491.

32. Jimeno D, Feiner L, Lillo C, Teofilo K, Goldstein LS, Pierce EA, Williams DS. Analysis of kinesin-2 function in photoreceptor cells using synchronous Cre-loxP knockout of Kif3a with RHOCre. Invest. Ophthalmol. Vis. Sci. 2006; 47: 5039-5046.

33. Tsujikawa M, Malicki J. Inflagellar transport genes are essential for differentiation and survival of vertebrate sensory neurons. Neuron 2004; 42: 703-716.

34. Krock BL, Perkins BD. The intraflagellar transport protein IFT57 is required for cilia maintenance and regulates IFT-kinesin-II dissociation in vertebrate photoreceptors. J. Cell Sci. 2008; 121: 19071915.

35. Sukumaran S, Perkins BD. Early defects in photoreceptor outer segment morphogenesis in zebrafish ift57, ift88 and ift172 intraflagellar transport mutants. Vis. Res. 2009; 49: 479-489.

36. Cao Y, Park A, Sun Z. Intraflagellar transport proteins are essential for cilia formation and for planar cell polarity. J. Am. Soc. Nephrol. 2010; 21: 1326-1333.

37. Huang P, Schier AF. Dampened hedgehog signalling but normal wnt signalling in zebrafish without cilia. Development 2009; 136: 3089-3098.

38. Trivedi D, Colin E, Louie CM, Williams D. Live-cell imaging evidence for the ciliary transport of rod photoreceptor opsin by heterotrimeric kinesin-2. J. Neurosci. 2012; 32: 10587-10593. 
39. Eblimit A, Nguyen TM, Chen Y, Esteve-Rudd J, Zhong H, Letteboer S, Van Reeuwijk J, Simons DL, Ding Q, Wu KM, Li Y, Van Beersum S, Moayedi Y, Xu H, Pickard P, Wang K, Gan L, Wu SM, Williams DS, Mardon G, Roepman R, Chen R. Spata7 is a retinal ciliopathy gene critical for correct RPGRIP1 localization and protein trafficking in the retina. Hum. Mol. Genet. 2015; 24: 1584-1601. 40. Louie CM, Caridi G, Lopes VS, Brancati F, Kispert A, Lancaster MA, Schlossman AM, Otto EA, Leitges M, Gröne HJ, Lopez I, Gudiseva HV, O'Toole JF, Vallespin E, Ayyagari R, Ayuso C, Cremers FP, den Hollander AI, Koenekoop RK, Dallapiccola B, Ghiggeri GM, Hildebrandt F, Valente EM, Williams DS, Gleeson JG. AHI1 is required for photoreceptor outer segment development and is a modifier for retinal degeneration in nephronophthisis. Nat. Genet. 2010; 42: $175-180$.

41. Alfinito PD, Townes-Anderson E. Activation of mislocalization opsin kills rod cells: a novel mechanism for rod cell death in retinal diseases. Proc. Natl. Acad. Sci. U. S. A. 2002; 99: 5655-5660. 42. Traverso V, Bush RA, Sieving PA, Deretic D. Retinal cAMP levels during the progression of retinal degeneration in rhodopsin $\mathrm{P} 23 \mathrm{H}$ and S334ter transgenic rats, Invest. Ophthalmol. Vis. Sci. 2002; 43: 1655-1661.

43. Tuma MC, Zill A, Le Bot N, Vernos I, Gelfand V. Heterotrimeric kinesin II is the microtubule motor protein responsible for pigment dispersion in Xenopus melanophores. J. Cell Biol. 1998; 143: $1547-1558$.

44. Deacon SW, Serpinskaya AS, Vaughan PS, Lopez Fanarraga M, Vernos I, Vaughan KT, Gelfand VI. Dynactin is required for bidirectional organelle transport. J. Cell Biol. 2003; 160: 297-301. 45. Kodani A, Sirerol-Piquer MS, Seol A, Garcia-Verdugo JM, Reiter JF. Kif3a interacts with Dynactin subunit p150Glued to organize centriole subdistal appendages. EMBO J. 2013; 32: 597-607. 


\section{Figure legends}

Figure 1 Expression of zebrafish Kif3a (ZFKif3A). (A) Temporal expression of ZFKif3a gene detected by RT-PCR from total RNA extracted from oocytes and embryos at different developmental stages. (B) ZFKif3a expression in different zebrafish adult tissues detected by RT-PCR.

Figure 2 Identification of zebrafish Kif3a mutation. (A) A schematic drawing of zebrafish Kif3a gene exon/intron structure. The red arrow points to the exon 9, which contains the mutation. (B) Sequences of wildtype (WT) and Kif3a ${ }^{\mathrm{SA} 1617}$ mutant allele. The arrow points to "C" in WT and "T" in the mutant. (C) The predicted truncated mutant polypeptide. (D) Localization of KIF3A protein in Kif $3 a^{S A 1617}$ mutant photoreceptors at $5 \mathrm{dpf}$ was absent. KIFA Anti-ac antibody labeled the connecting cilia of photoreceptors.

Figure 3 External phenotypes of wildtype (WT, left panel) and Kif $3 a^{S A 1617}$ mutants (right panel) of different ages. The mutant zebrafish displayed curved body axis, smaller eyes, cyst formation and abnormal swim bladder. Blue arrow indicates kidney cyst while yellow arrow indicates the swim bladder.

Figure 4 Abnormal outer segment development in $\mathrm{Kif}_{3} \mathrm{a}^{\mathrm{SA1617}}$ mutants (MUT) at $4 \mathrm{dpf}$ (A) and $5 \mathrm{dpf}$ (B). The wildtype (WT) siblings exhibited normal outer segment formation (A-1 and B-1). Outer segments were not formed in most parts of mutant retinas (A-2 and B-2). In peripheral retina, some abnormal outer segments were formed. A-3, distended outer segment with folded protrusion; A-4, annular outer segment; A-5, irregular branching outer segment; A-6, compressed and distended outer segment; B-3, vestigial outer segment; B-4, vestigial and annular outer segment; B-5, annular and delaminated outer segment; B-6, warped outer segment with irregular delamination. M, mitochondria; MEL, melanosome; N, nuclear; OS, outer segment.

Figure 5 Retinal histology of wildtype (WT) and Kif3a ${ }^{\mathrm{SA} 1617}$ mutant zebrafish at 3, 5, 6 and 9 dpf. Haematoxylin and eosin staining of WT and mutant retinal sections showing rapid retinal 
degeneration. A yellow line indicates the boundary of outer nuclear and inner nuclear layers. GCL, ganglion cell layer; INL, inner nuclear layer; ONL, outer nuclear layer; RPE, retinal pigment epithelium.

Figure 6 Retinal cryosection of wildtype (WT) and Kif $3 a^{S A 1617}$ mutant larvae at 5 dpf were immunostained with anti-rhodopsin (4D2) antibody to label rod outer segments (A) and with ZPR1 antibody to label red/green double cones (B). Outer segments were not evident in the mutants. The rhodopsin was mislocalized in rod cell bodies. Cone cells were degenerated.

Figure 7 TUNEL assay showed cell death in Kif3a ${ }^{\mathrm{SA} 1617}$ mutant retinas at $5 \mathrm{dpf}$. In the mutants TUNEL-positive cells were present in the outer nuclear layer (ONL) of both central and peripheral retinas. TUNEL-positive cells were barely observed in ONL of wildtype retina (A). (B) Graph indicating significant cell death in the mutant retina compared to the wildtype retina. $* * * p<0.001$.

Figure 8 Loss of Kif3a results in caffeine-induced melanosome dispersion delay. (A) Control and $\mathrm{Kif3a}^{\mathrm{SA} 1617}$ mutant larvae prior to epinephrine treatment, after 2 min of epinephrine exposure, the endpoint after continuous epinephrine exposure, and the recovery with caffeine exposure. (B) Graphical representation demonstrating a significant delay in caffeine induced melanosome anterograde trafficking for Kif3 $\mathrm{a}^{\mathrm{SA} 1617}$ mutants ( $\mathrm{p}<0.0001$ by $t$-test) when compared with the controls. However there was no difference for melanosome retrograde trafficking when compared with the controls $(\mathrm{p}=0.6721) . * * * * \mathrm{p}<0.0001$.

\section{Supplementary Figure legends}

Figure S1 Alignment of Kif3a protein sequences from Zebrafish (NP_001017604), human (NP_001287720), mouse (NP_032469), Xenopus (XP_002935308.1), chicken (NP_001025793) and C.elegans (NP_497178). 
Figure S2 Kif3a expression was significantly reduced in Kif3 $\mathrm{A}^{\mathrm{SA} 1617}$ mutants possibly through nonsense mediated mRNA decay. The relative expression of Kif $3 A$ in wildtype and Kif $3 a^{\text {SAl617 }}$ mutant zebrafish at $5 \mathrm{dpf}$ was measured by quantitative real-time PCR and normalized to $\beta$-actin. ${ }^{* *} \mathrm{p}<0.01$.

Figure S3 Kif3a ${ }^{\mathrm{SA} 1617}$ mutant zebrafish have smaller eyes than that of wildtype (WT) siblings. The diameter (eye size) of the eyes from wildtype and Kif3 $\mathrm{A}^{\mathrm{SA} 1617}$ mutant zebrafish at 2, 3, 4, 5, and $6 \mathrm{dpf}$ was measured and the data were subjected to statistical analysis. ns, no significance; ${ }^{* *} \mathrm{p}<0.01 ; * * *$ $\mathrm{p}<0.001 ; * * * * \mathrm{p}<0.0001$.

Figure S4 No electroretinography (ERG) response in $\mathrm{Kif}_{3} a^{\text {SAl617 }}$ mutant zebrafish. 7 dpf wildtype $(\mathrm{n}=10)$ and $\mathrm{Kif}^{\mathrm{S}} \mathrm{a}^{\mathrm{S} 1617}$ mutant zebrafish $(\mathrm{n}=8)$ were subjected to ERG test under photopic condition, wildtype exhibited normal ERG response but the response was absent in Kif3 ${ }^{\text {SAl617 }}$ mutant zebrafish. 


\section{Figure 1}
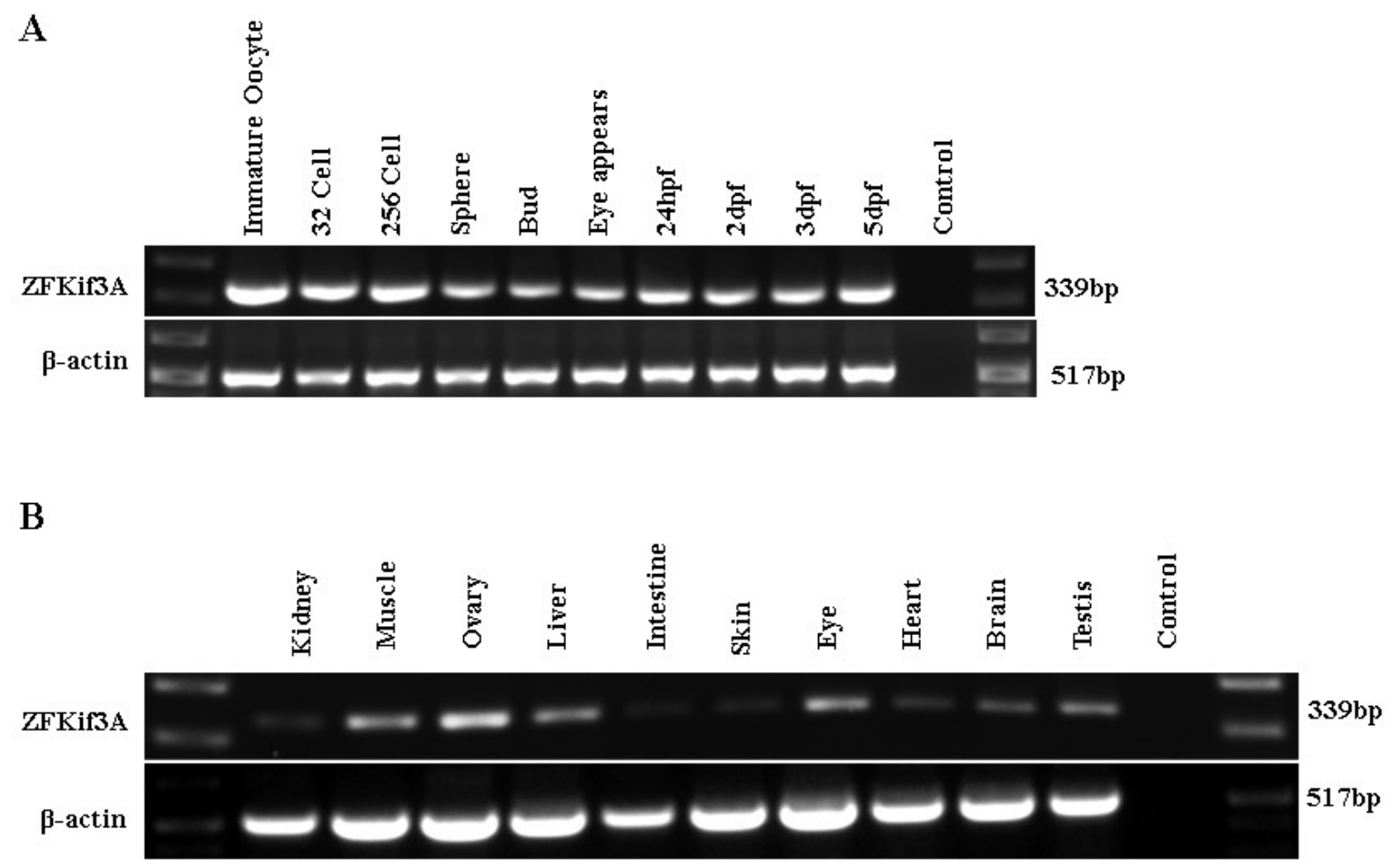


\section{Figure 2}

A

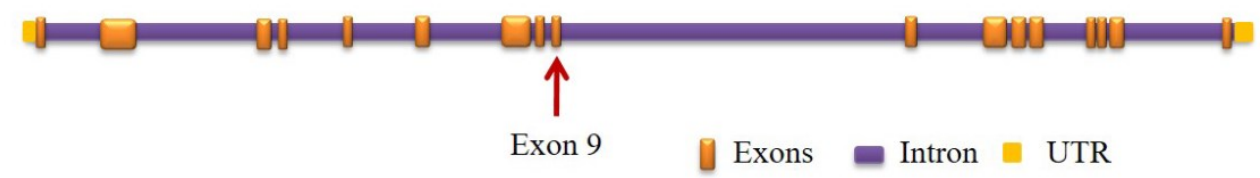

B
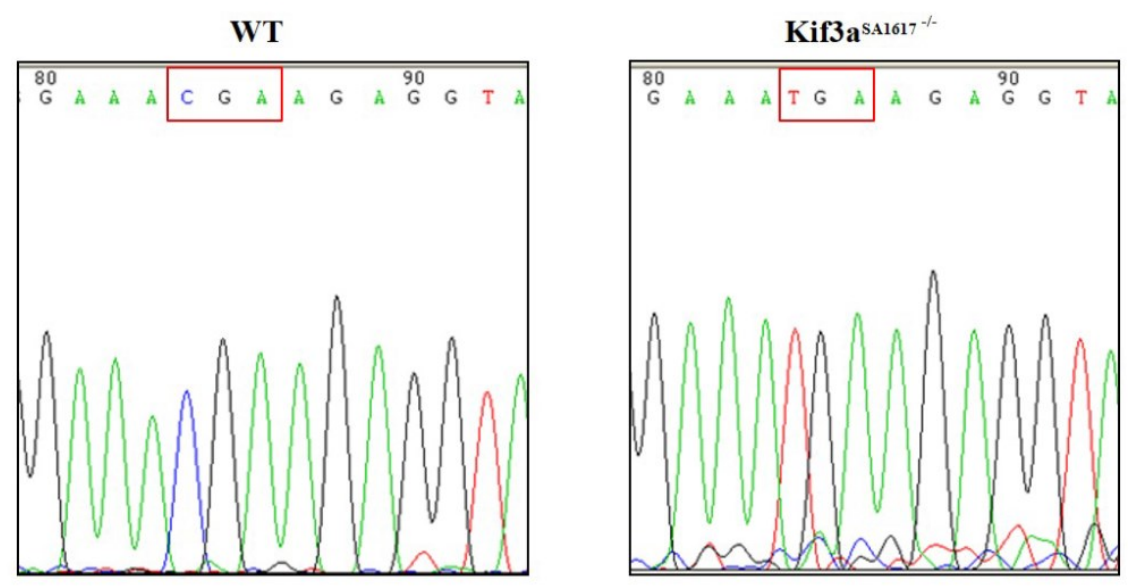

C

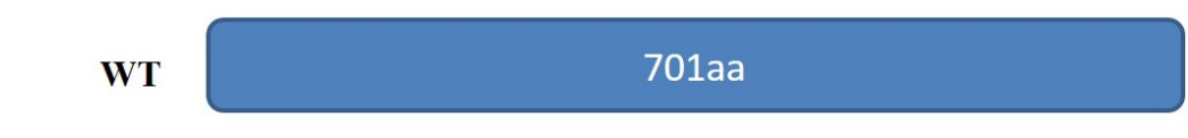

Kif3a $^{\mathrm{SA1617} 7^{-1}}$

454aa

D

WT

Kif3a ${ }^{\mathrm{SA} 1617^{-/}}$

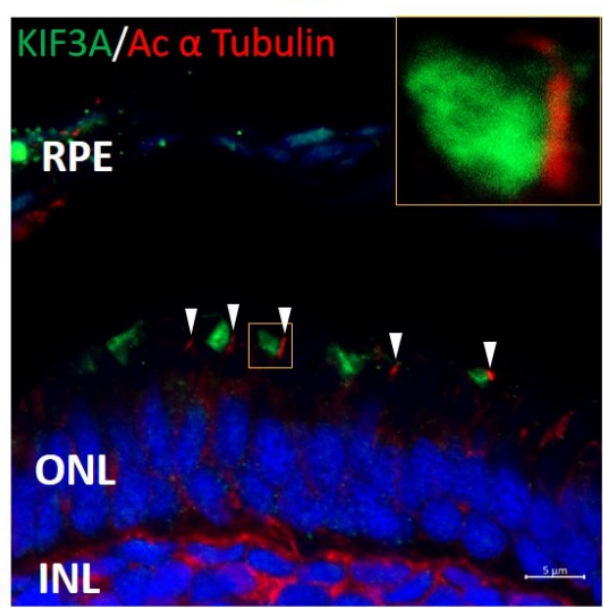

RPE

ONL

INL 
Figure 3

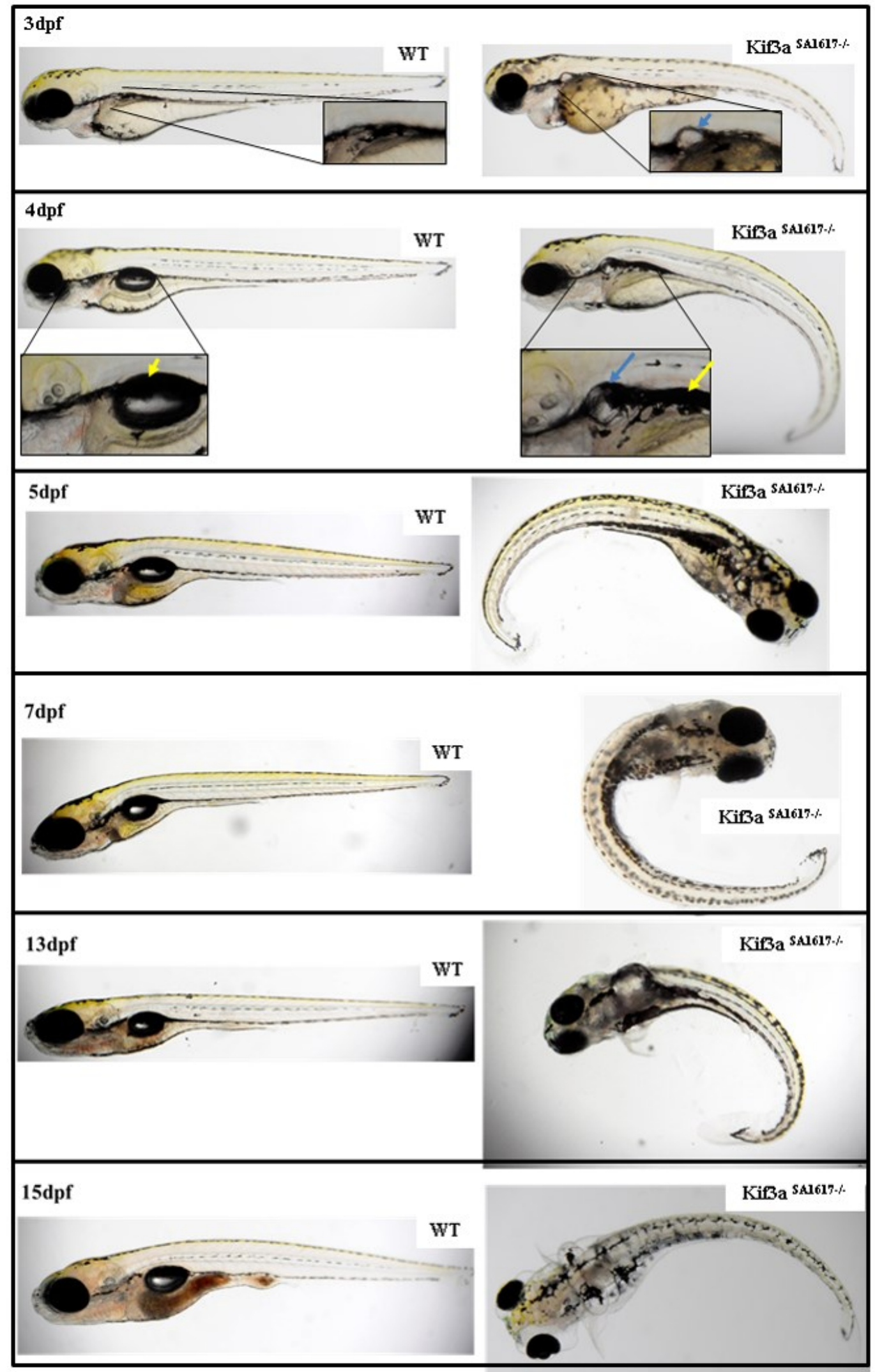


Figure 4

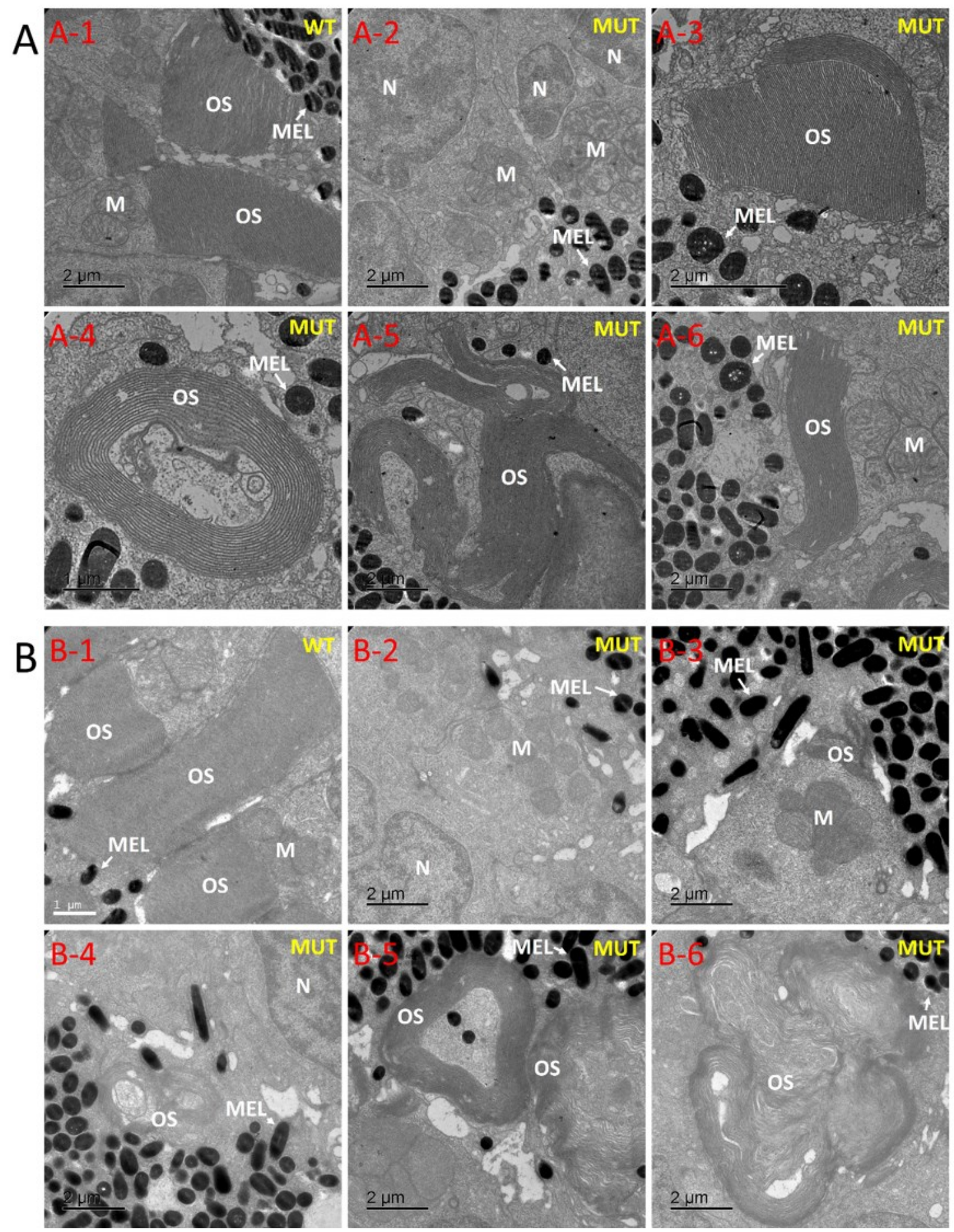


Figure 5

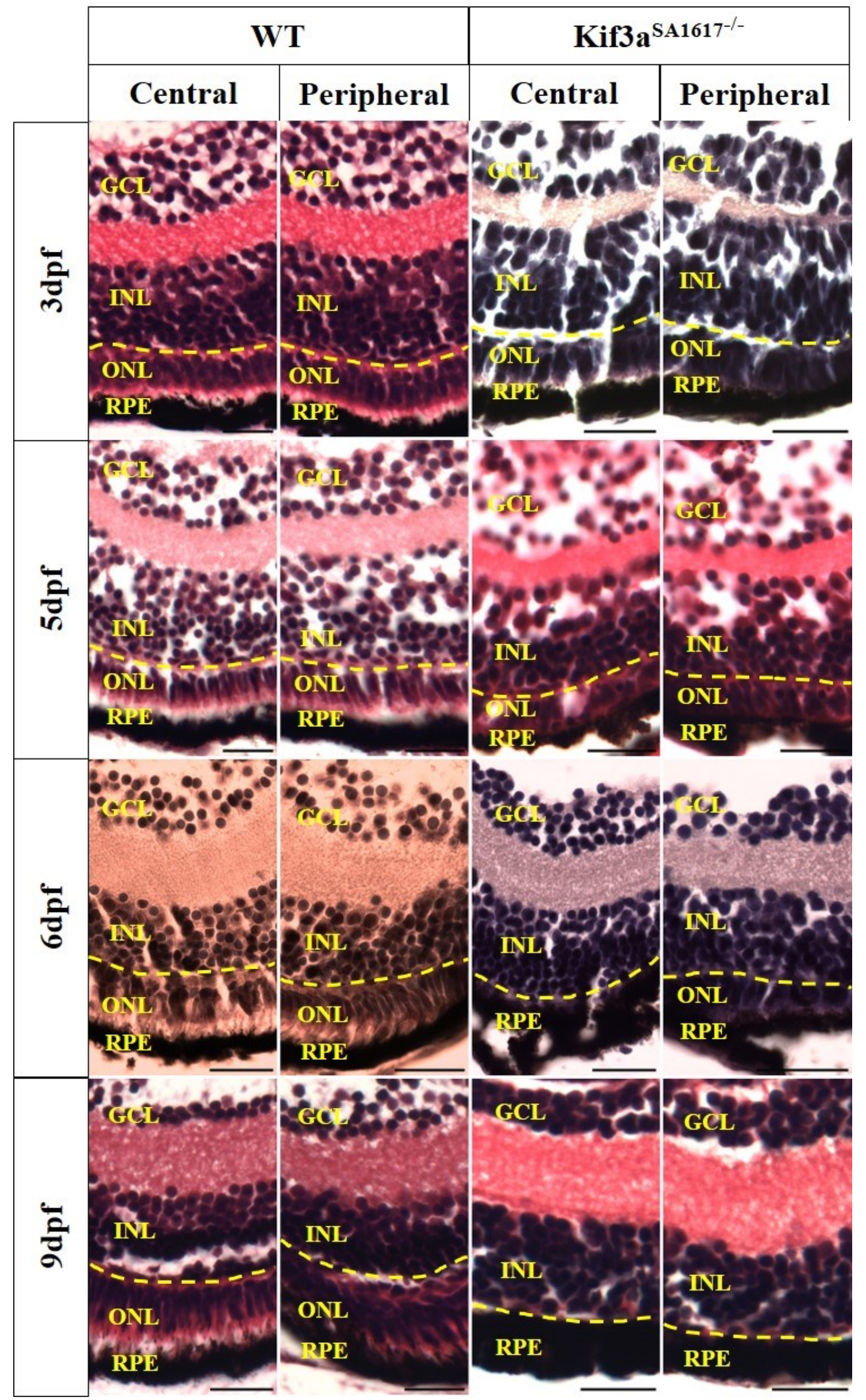


Figure 6

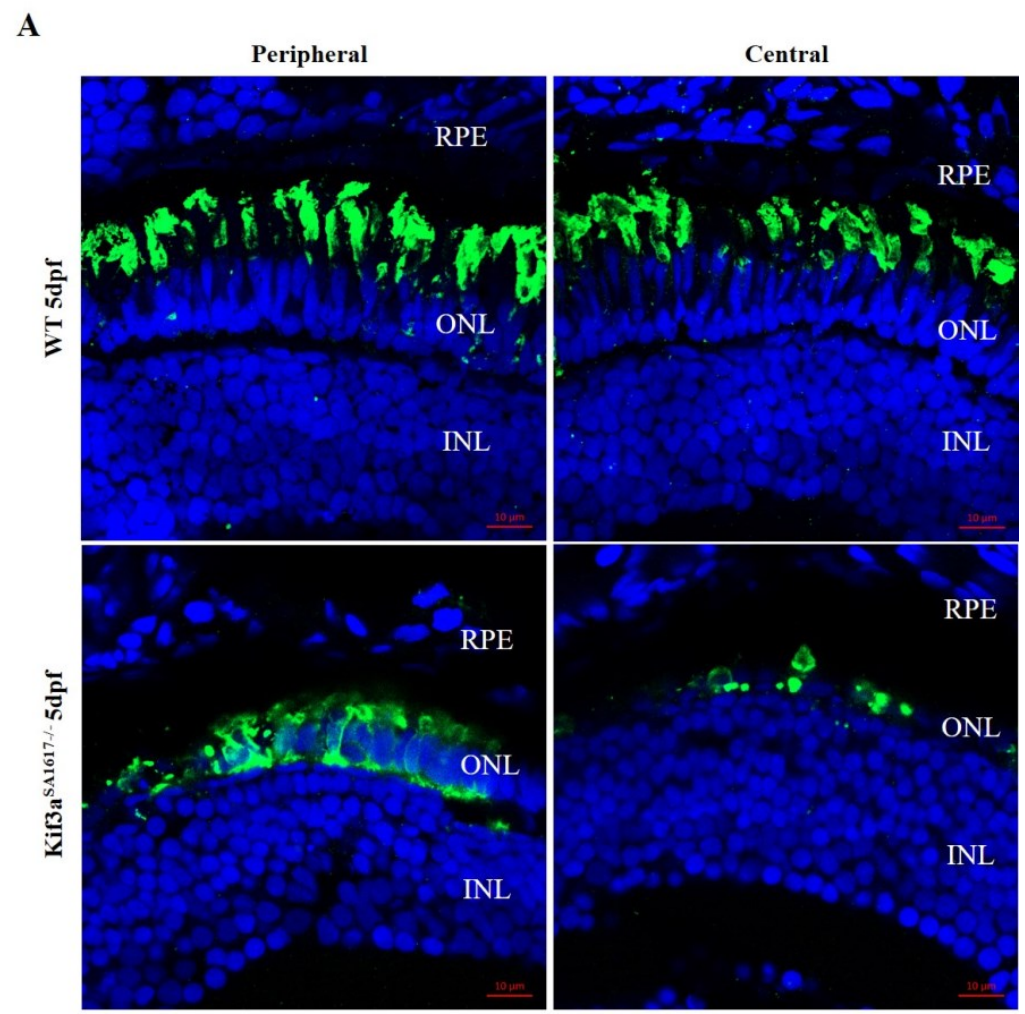

B

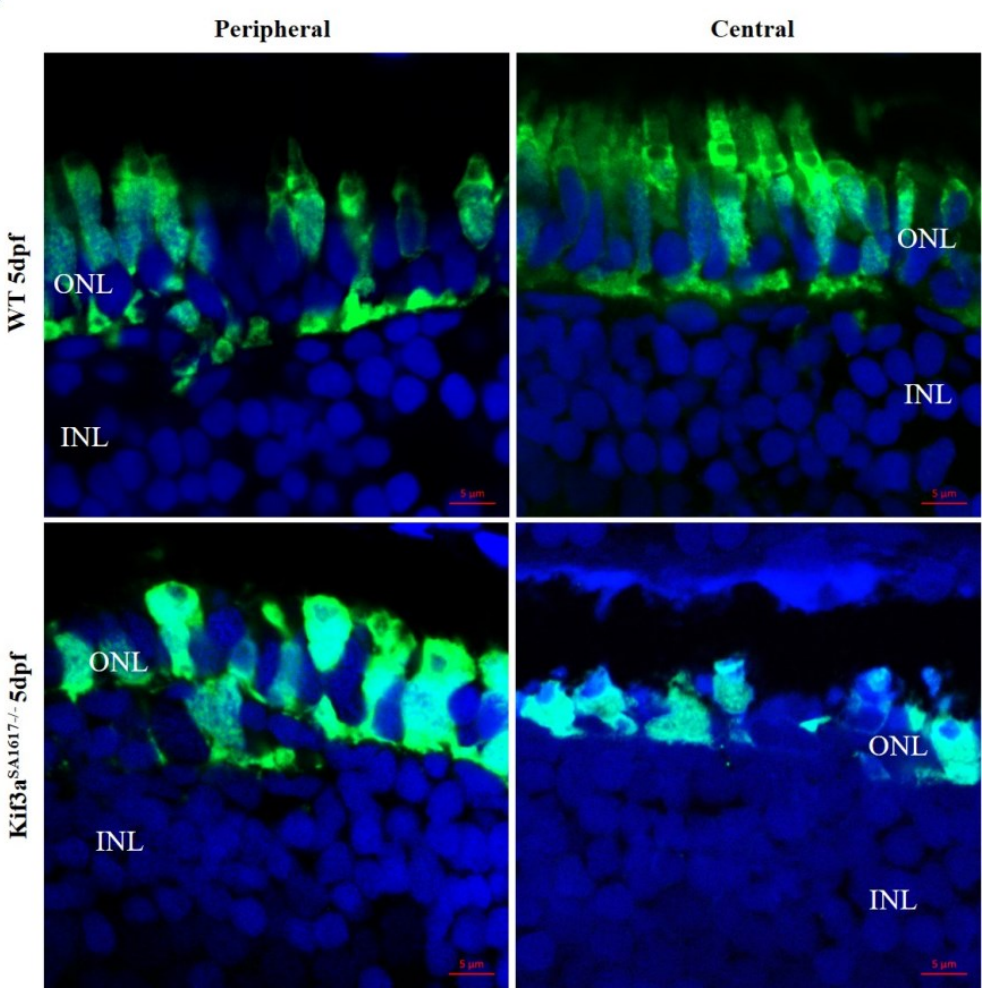


Figure 7

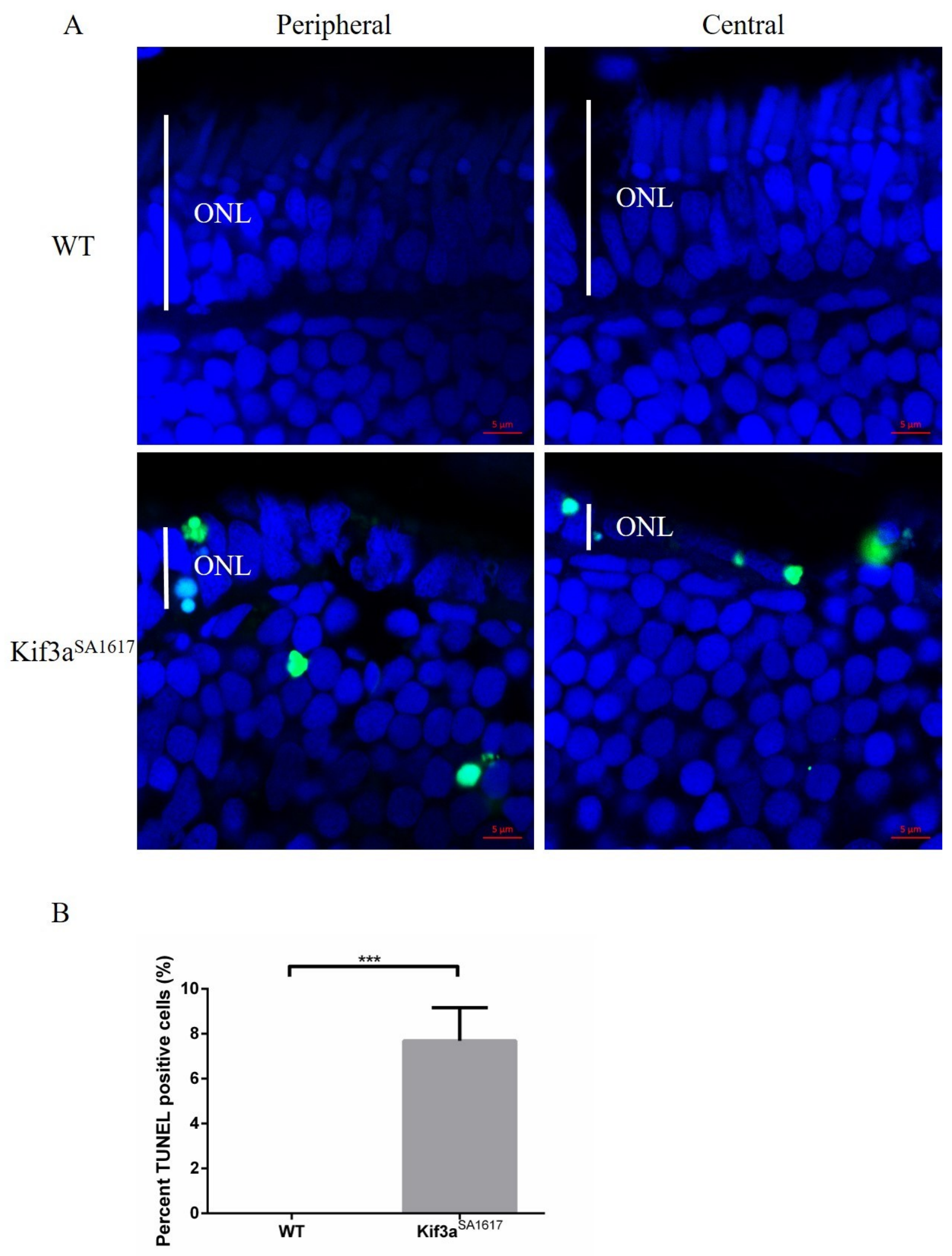


Figure 8

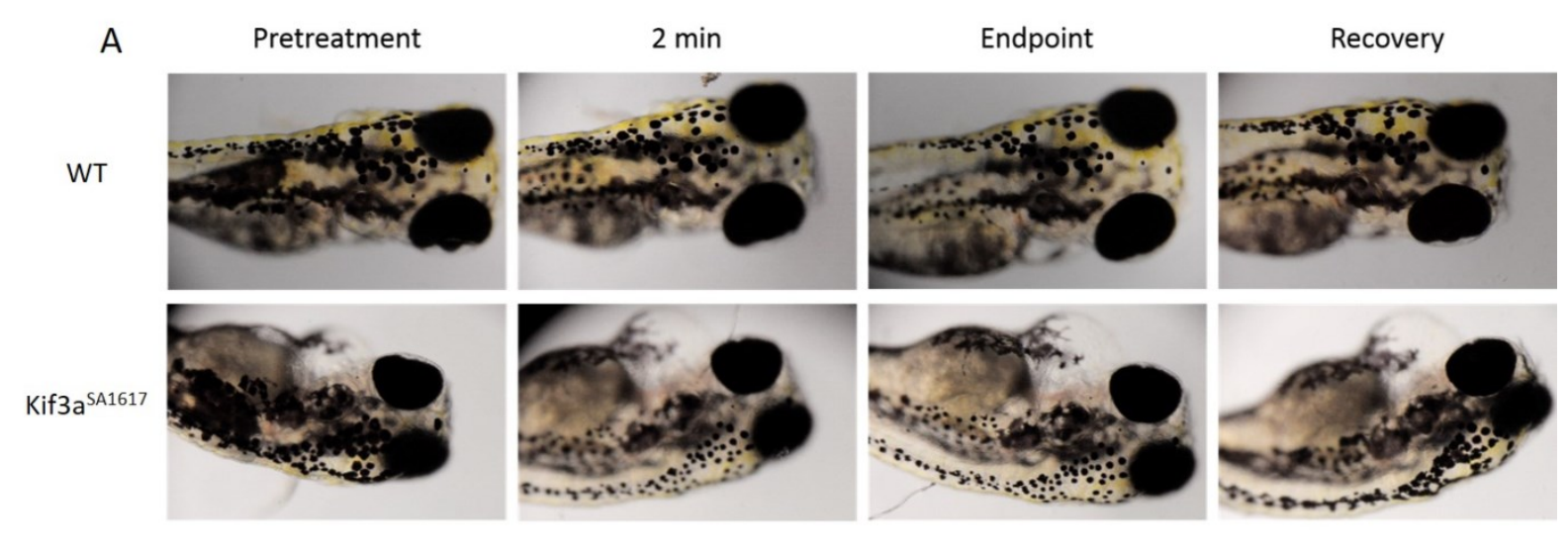

B

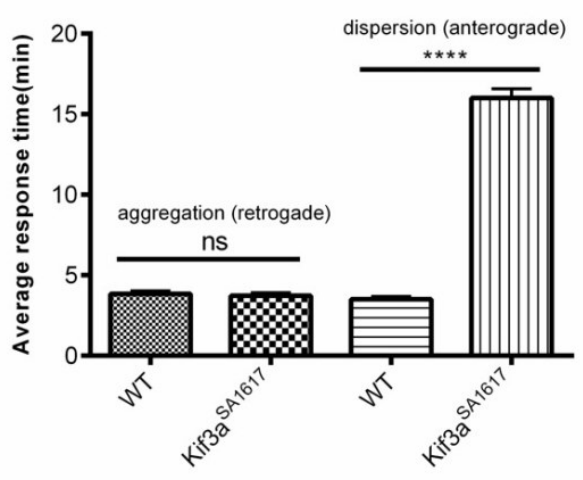


Supplementary Figures

Figure S1

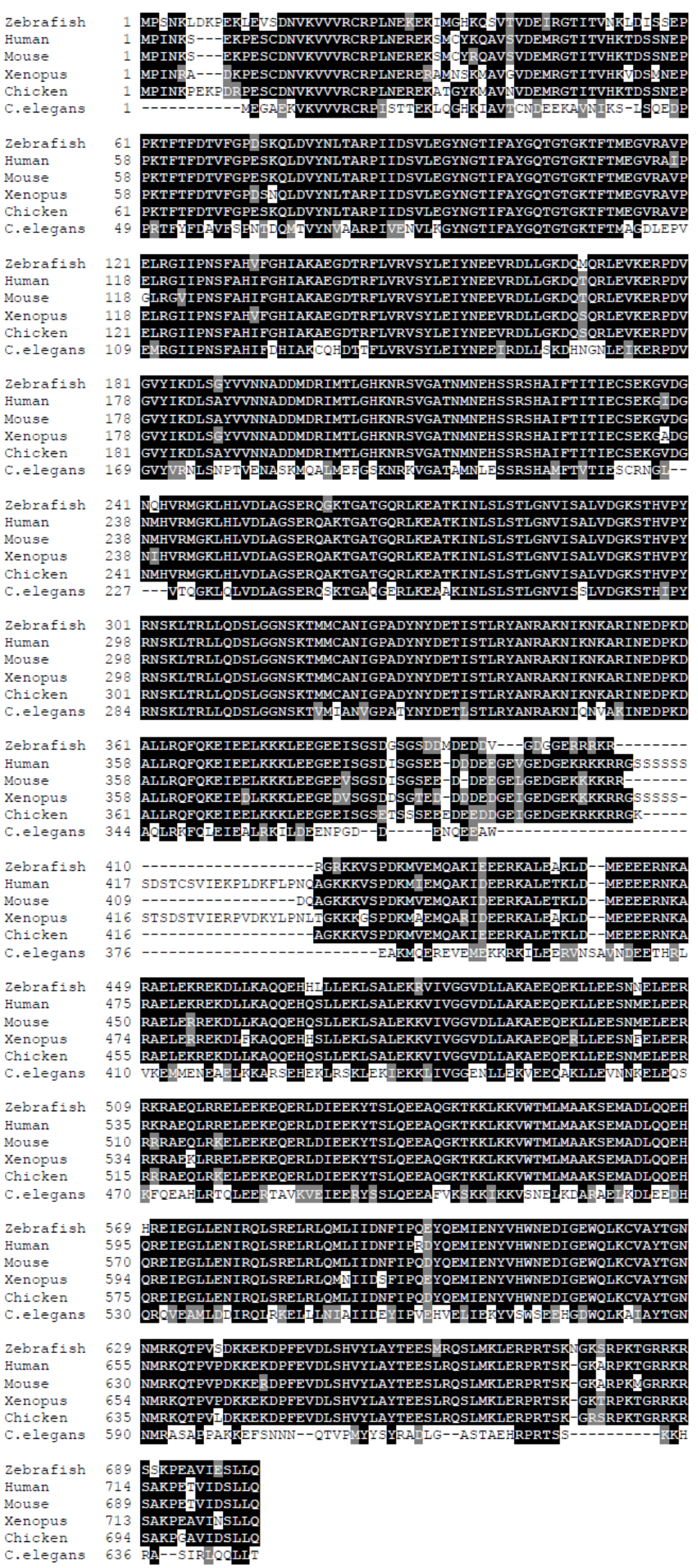


Figure S2

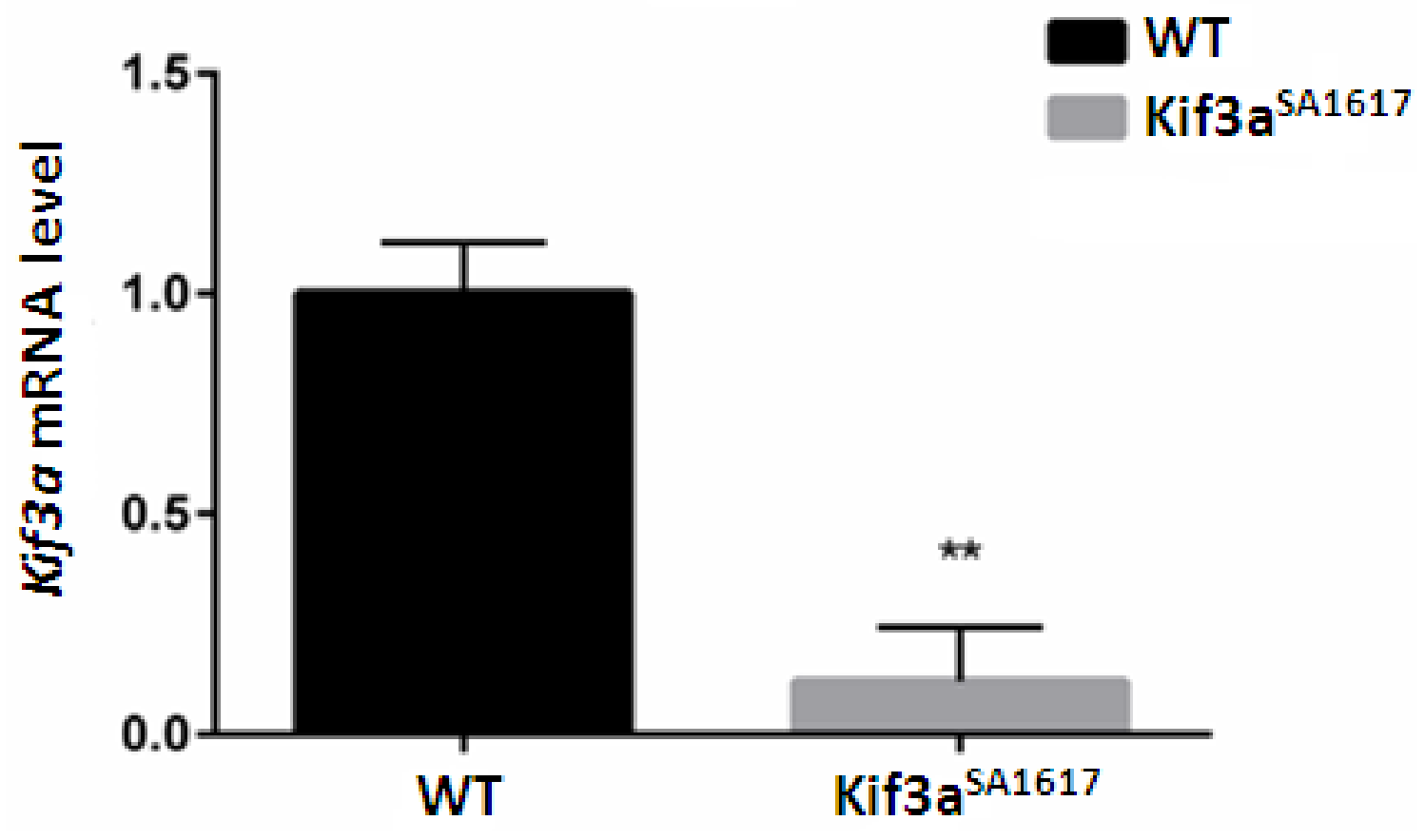

Figure S3

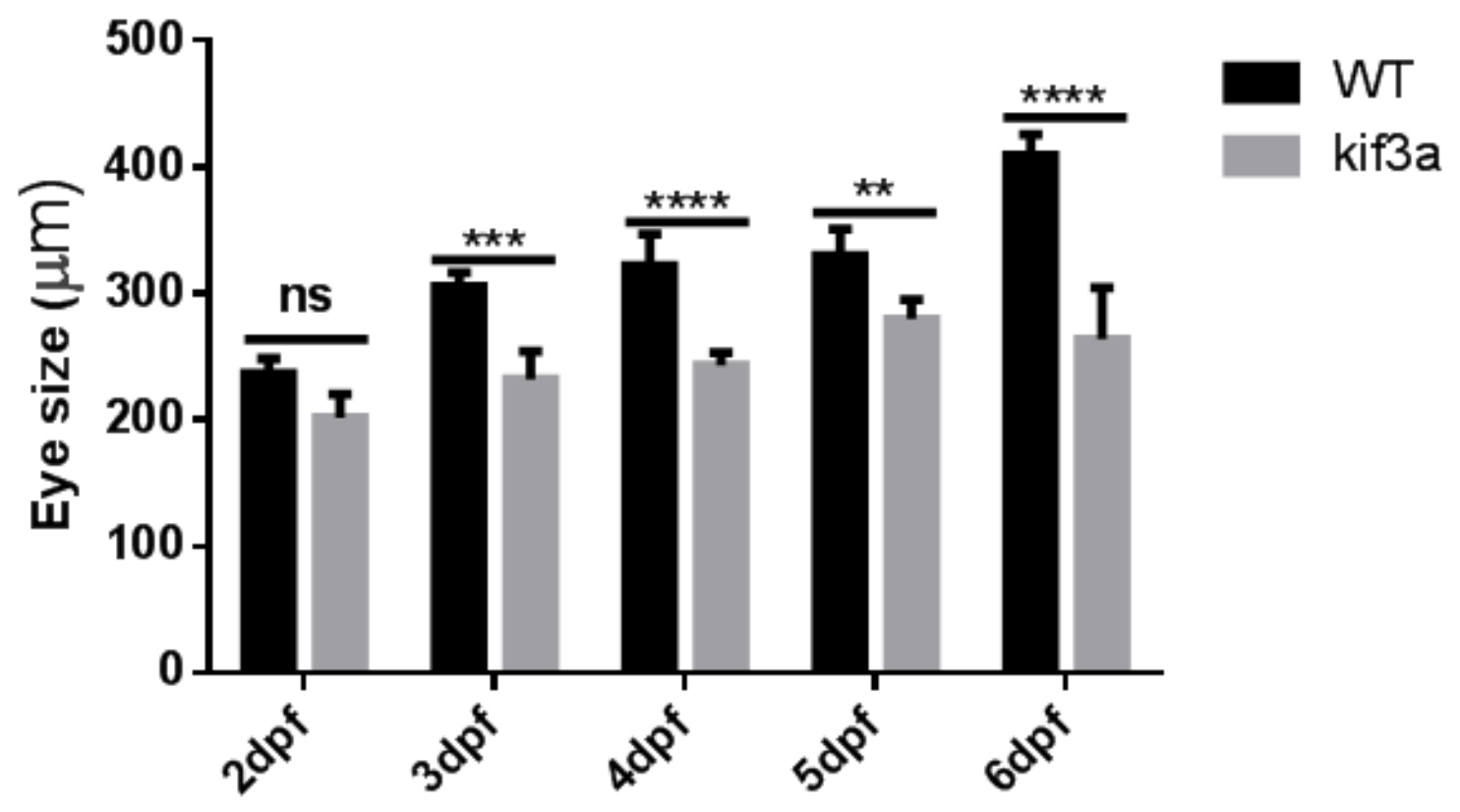


Figure S4

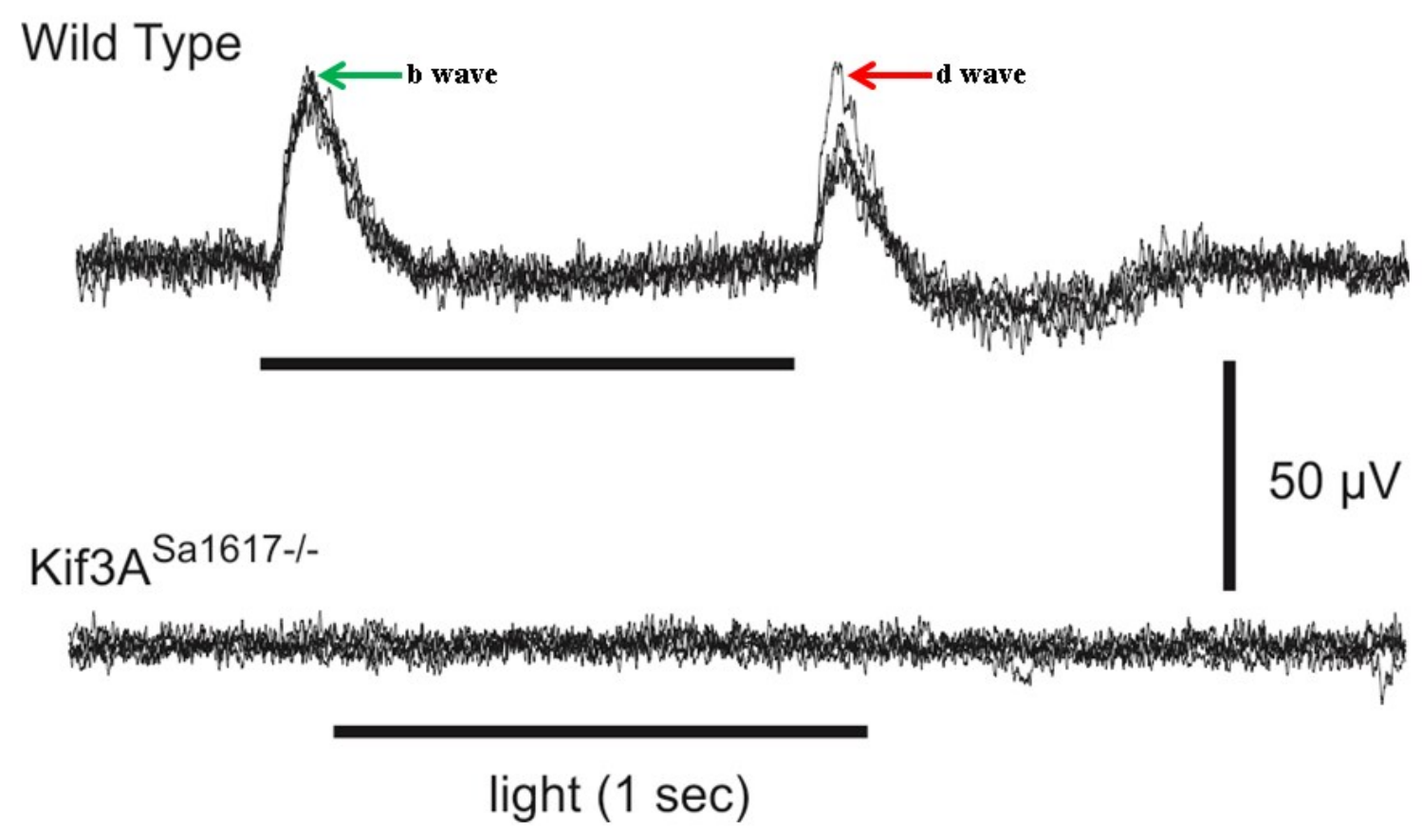

